\title{
High Glucose Accelerates Autophagy in Adult Rat Intervertebral Disc Cells
}

\author{
Chae-Gwan Kong, Jong-Beom Park, Man Soo Kim, Eun-Young Park \\ Department of Orthopedic Surgery, Uijeongbu St. Mary's Hospital, The Catholic University of Korea College of Medicine, Korea
}

\begin{abstract}
Study Design: In vitro cell culture.
Purpose: The purpose of this study was to investigate the effect of high glucose on autophagy in adult rat intervertebral disc cells. Overview of Literature: Diabetes mellitus is considered to be an important etiologic factor for intervertebral disc degeneration, resulting in degenerative disc diseases. A glucose-mediated increase of autophagy is a major causative factor for the development of diseases associated with diabetes mellitus. However, no information is available for the effect of high glucose on autophagy in adult intervertebral disc cells.

Methods: Nucleus pulposus and annulus fibrosus cells were isolated from 24-week-old adult rats, cultured and placed in either 10\% fetal bovine serum (normal control) or 10\% fetal bovine serum plus two different high glucose concentrations (0.1 M and $0.2 \mathrm{M}$ ) (experimental conditions) for one and three days, respectively. The expressions of autophagy markers, such as beclin-1, light chain 3-I (LC3-I) and LC3-II, autophagy-related gene (Atg) 3, 5, 7 and 12, were identified and quantified.

Results: Two high glucoses significantly increased the expressions of beclin-1, LC3-II, Atg3, 5, 7, and 12 in adult rat nucleus pulposus and annulus fibrosus cells in a dose- and time-dependent manner. The ratio of LC3-II/LC3-I expression was also increased in a doserespectively time-dependent manner.

Conclusions: The results suggest that autophagy of adult nucleus pulposus and annulus fibrosus cells might be a potential mechanism for the intervertebral disc degeneration in adult patients with diabetes mellitus. Thus, the prevention of autophagy in adult intervertebral disc cells might be considered as a novel therapeutic target to prevent or to delay the intervertebral disc degeneration in adult patients with diabetes mellitus.
\end{abstract}

Keywords: Diabetes mellitus; High glucose; Autophagy; Adult intervertebral disc cells; Intervertebral disc degeneration

\section{Introduction}

Diabetes mellitus (DM) is a major public health problem worldwide. There are two main types of DM. Type $1 \mathrm{DM}$ results from the body's failure to produce insulin and is referred to as "insulin-dependent DM (IDDM)" or "juvenile-onset DM". Type 2 DM results from insulin resistance and is referred to as "non-insulin-dependent
DM (NIDDM)" or "adult-onset DM". Approximately 90\% of all cases of DM are Type 2 DM. The number of DM patients will reach 300 million in 2025 (International Diabetes Federation, 2001) [1]. DM prevalence increases with age, and the number of older persons with DM are expected to grow as the elderly population increases in number. Untreated DM can cause many complications, such as cardiovascular disease, chronic renal failure,

Received Jan 25, 2014; Revised Jul 29, 2014; Accepted Aug 27, 2014

Corresponding author: Jong-Beom Park

Department of Orthopedic Surgery, Uijeongbu St. Mary's Hospital, The Catholic University of Korea College of Medicine,

271 Cheonbo-ro, Uijeongbu 480-717, Korea

Tel: +82-31-820-3578, Fax: +82-31-847-3671, E-mail: spinepjb@catholic.ac.kr 
retinopathy, and neuropathy [2]. DM, especially type 2 or adult-onset, is known to be an important etiological factor of disc degeneration, resulting in degenerative disc diseases [3-7]. Hyperglycemia is a major causative factor in the development of diabetes mellitus-associated diseases through the excessive generation of reactive oxygen species (ROS) [6]. ROS are thought to play a role in varied cellular processes, such as apoptosis, senescence and autophagy, which cause cellular damage and lack a physiological function [8-11].

Autophagy is a physiologic mechanism in which cells degrade unnecessary or dysfunctional cellular organelles through the lysosomal machinery [12]. A proper regulation of autophagy guarantees the fine balance of synthesis, degradation and recycling of cellular components. These functions render autophagy which is essential for the proper cell differentiation and organ development. Autophagy consists of multi-step processes that are highly regulated by autophagy-related genes (Atgs) and light chain protein 3 (LC3) [13-15]. During autophagy, the cytosolic microtubule-associated protein LC3-I is lipidated and converted to LC3-II, which is translocated to the autophagosomal membrane. Thus, the conversion of LC3-I to LC3-II and accumulation of LC3 are widely used as markers of autophagy. Beclin-1 is a $\mathrm{BH} 3$ member of the Bcl-2 gene family that can drive the autophagic process in mammalian cells $[16,17]$. Although autophagy is generally viewed as a survival mechanism, excessive or dysregulated autophagy has recently been implicated in the pathogenesis of various diseases including cancer, neurodegenerative and cardiovascular diseases $[15,18$ 21].

Jiang et al. [22] suggested autophagy may be a response mechanism to the apoptotic and senescent changes of young notochordal cells which were caused by injection of strepzotocin in young diabetic rats. Park et al. [23] also reported that high glucose-induced oxidative stress promotes the autophagy through mitochondrial damage of young notochordal cells in young rat. However, two previous studies investigated the autophagy limited to young notochordal cells. Thus, no information was available about the effect of diabetes mellitus on the autophagy in adult NP and AF cells. Therefore, the authors performed the current study to investigate the effect of high glucose on autophagy in adult rat nucleus pulposus (NP) and annulus fibrosus (AF) cells in an in vitro cell culture model.

\section{Materials and Methods}

\section{Intervertebral disc cell culture and treatment with two different high glucose concentrations}

All lumbar intervertebral discs (L1-6) were harvested from 24-week-old male Sprague Dawley rats (Orient Bio., Seoul, Korea) immediately after they were sacrificed. To separately obtain NP and AF tissues, the discs were carefully dissected under a microscope and the harvested NP and AF tissues were pooled in [alpha]-minimum essential medium ([alpha]-MEM, Gibco BRL, Grand Island, NY, USA). The cells were released from the NP and AF tissues by vigorous pipetting in HBSS (Hyclone, Ottawa, Ontario, Canada) with $0.02 \%$ pronase (Sigma-Aldrich, St. Louis, MO, USA). The cells were cultured in a complete medium [alpha]-MEM supplemented with $10 \%$ fetal bovine serum (FBS; Hyclone) and 1\% penicillin-streptomycin (Gibco BRL) at $37^{\circ} \mathrm{C}$ in a humidified atmosphere (95\% air, $-5 \% \mathrm{CO}_{2}$ ). After the cells grew to confluence, they were split once (passage 1) and grew to confluence again. Then the cells were trypsinized, washed and plated onto 6- or 12-well culture plates. When the cells reached 80 to $90 \%$ confluence, the NP and AF cells were placed in either FBS for normal control or in 10\% FBS plus 2 different high glucose concentrations $(0.1 \mathrm{M}$ and $0.2 \mathrm{M})$ for experimental conditions for 1 and 3 days. This study was approved by the Animal Care and Use Committee of the institution.

\section{Expressions and activities of autophagy makers}

For the western blot analysis of autophagy markers, primary antibodies to beclin-1, LC3-I and LC3-II, and Atg 3, 5, 7, and 12 were purchased from Cell Signaling Technology Inc. (Danvers, MA, USA). In addition, the antibody for loading control $\mathrm{B}$-actin was obtained from SIGMA (Sigma, St. Louis, MO, USA). Expressions of beclin-1, LC3-I and -II, and Atg3, 5, 7 and 12 were determined by western blot analysis according to the manufacturer's instructions. $B$-actin was used as an internal control for protein-loading.

$\mathrm{NP}$ and AF cells were washed with ice-cold phosphate buffer saline (PBS) solution and lysed in PRO-PREP protein extraction solution (iNtRON Biotechnology Inc., Sungnam, Korea). The cell lysates were centrifuged at $12,000 \mathrm{~g}$ for 15 minutes and protein concentrations were 
measured using the Bicinchoninic Acid (BCA) (Thermo Fisher Scientific Inc., Pittsburgh, PA, USA) method. Samples (50 to $70 \mu \mathrm{g}$ of protein) were electrophoresed on $10 \%$ to $15 \%$ sodium dodecyl sulfate-polyacrylamide gel electrophoresis (SDS-PAGE) and transferred to a nitrocellulose membrane. The membranes were incubated with primary antibodies, followed by second antibodies of HRP (horseradish peroxidase)-linked immunoglobulin G (Bio-Rad, Richmond, CA, USA). The immunoreactive bands were visualized with the western blotting luminol reagent (Santa Cruz Biotechnology Inc., Santa Cruz, CA, USA). Blots were quantified three times in each sample using Imaging Densitometer GF670 and Molecular Analyst software (Bio-Rad). The average of the three densities was used as the final density. The value of the density is presented as mean \pm standard deviation (arbitrary units).

\section{Statistical analysis}

All experiments were independently conducted three times and the results are expressed as mean and standard deviation. Statistical analysis was performed using a paired-samples $t$ test. $p<0.05$ was considered significant.

\section{Results}

\section{Expression of autophagy markers in the adult rat NP cells}

The western blot analysis showed a dose- and timedependent increased expression of beclin-1, LC3-II and Atg3, 5, 7 and 12 in the NP cells of 24-week-old rats treated with both high glucose concentrations when compared to the control (Fig. 1A, B). The ratio of LC3II/LC3-I expression also increased in a dose- and timedependent manner (Fig. 1C).

\section{Expression of autophagy markers in adult rat AF cells}

The western blot analysis showed a dose- and timedependent increased expression of beclin-1, LC3-II and Atg3, 5, 7 and 12 in the AF cells of 24-week-old rats treated with both high glucose concentrations when compared to the control (Fig. 2A, B). The ratio of LC3II/LC3-I expression also increased in a dose- and timedependent manner (Fig. 2C).
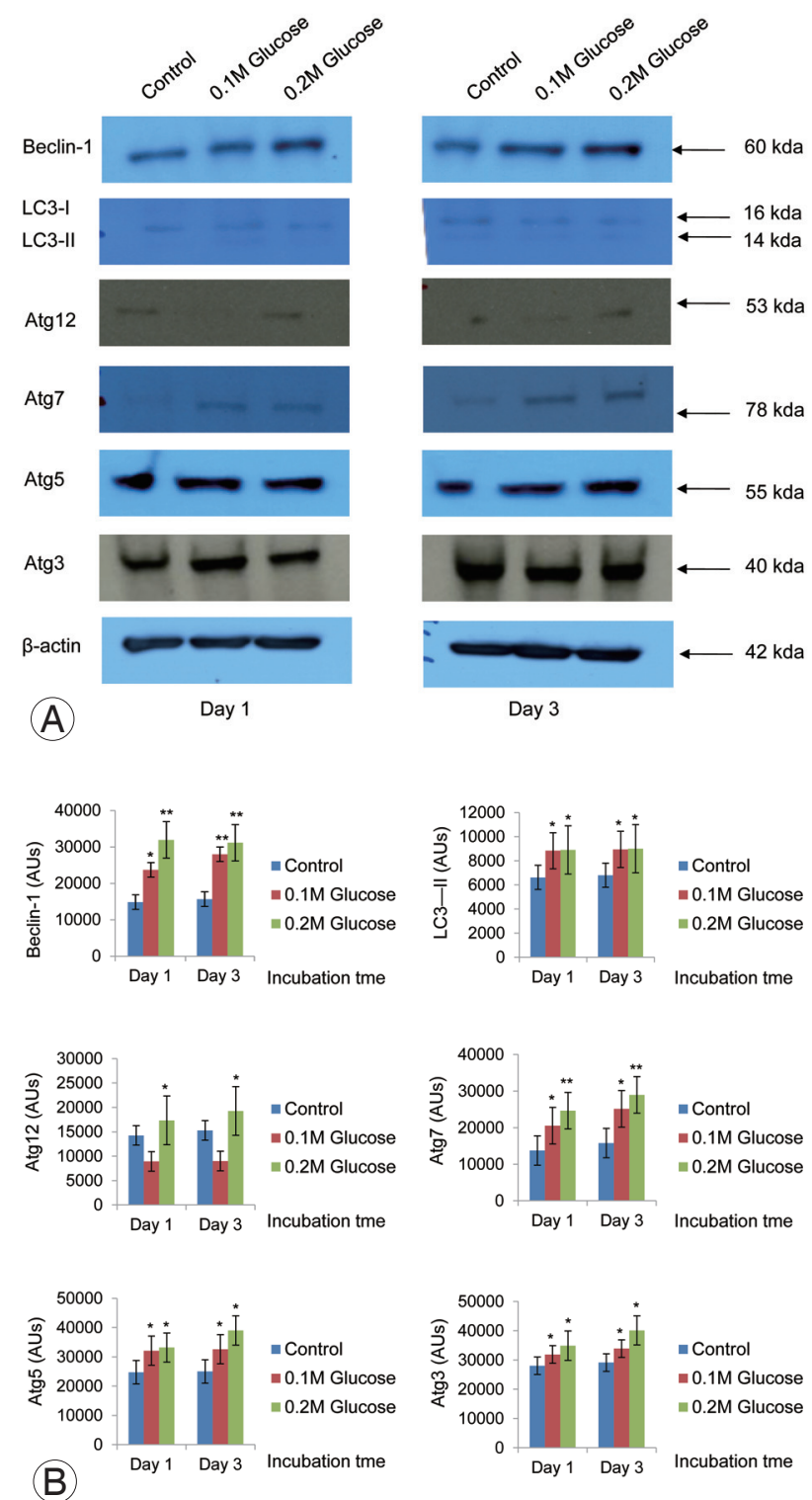

(C)

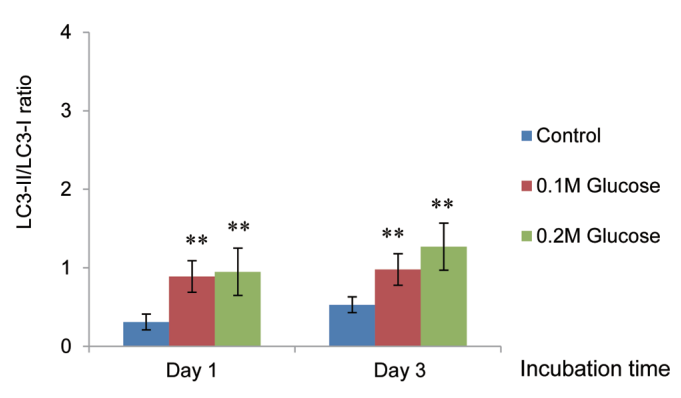

Fig. 1. The Western blot analysis shows a dose- and time-dependent increased expression of beclin-1, LC3-II and Atg3, 5, 7, and 12 in adult rat nucleus pulposus cells treated with both high glucose concentrations compared to the control (A, B). The ratio of LC3-II/LC3-I expression also increased in a dose- and time-dependent manner (C). LC, light chain; Atg, autophagy-related gene. ${ }^{*} p<0.05 ;{ }^{* *} p<0.01$; ${ }^{* * *} p<0.001$. 

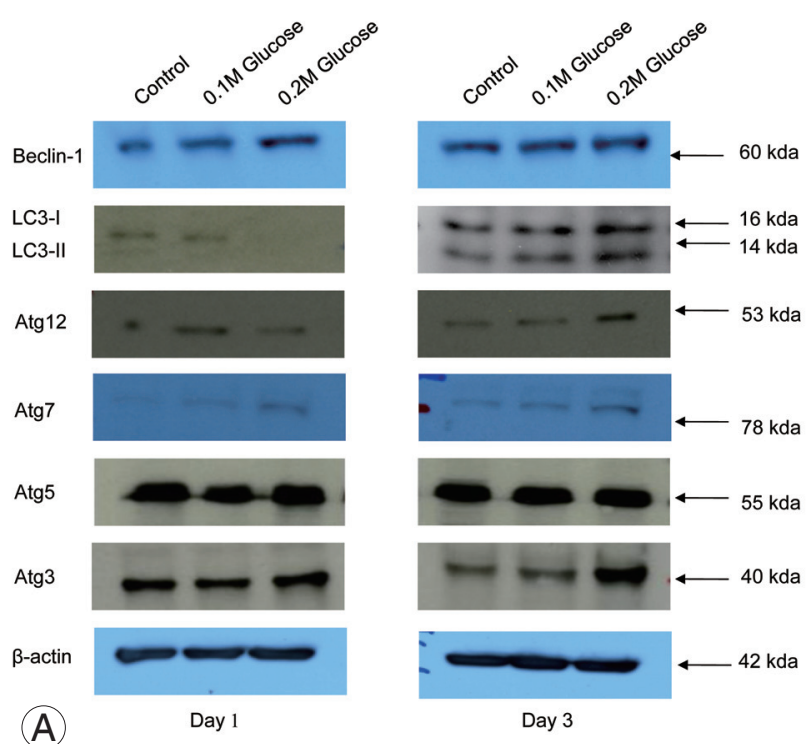

(A)
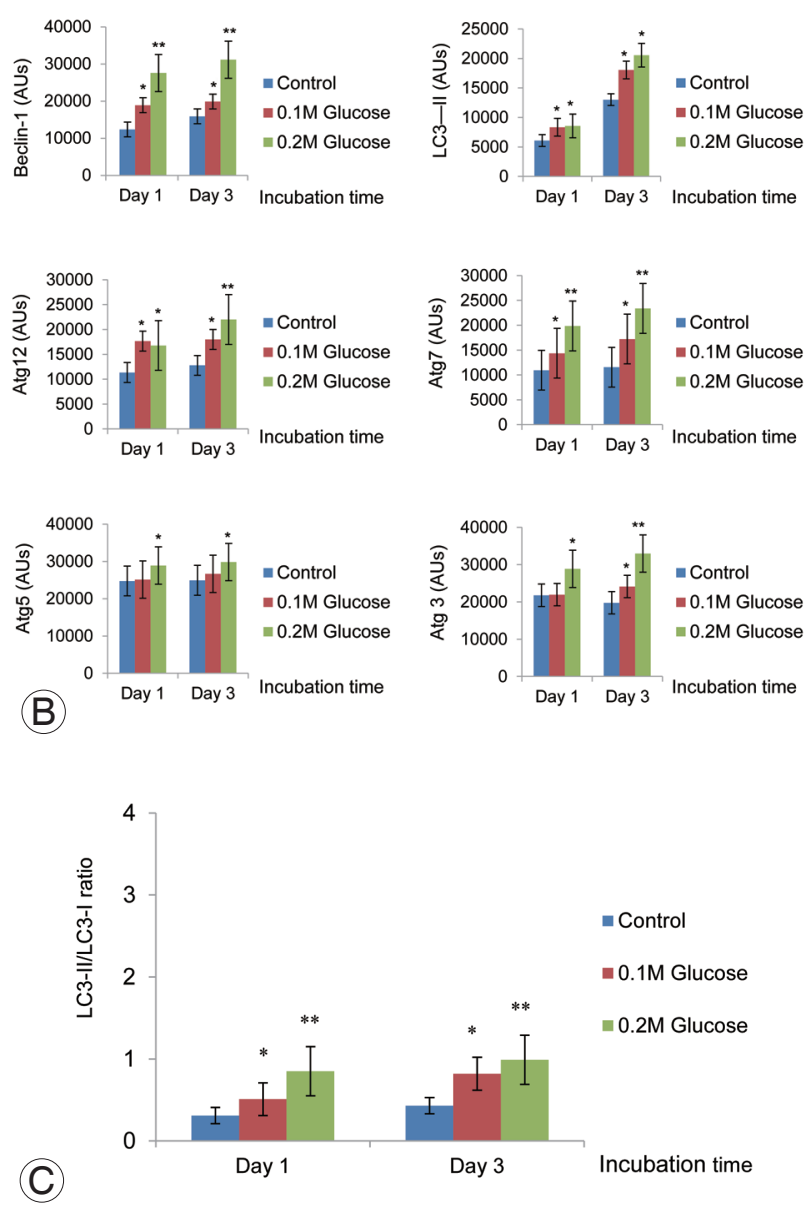

Fig. 2. The Western blot analysis shows a dose- and time-dependent increased expression of beclin-1, LC3-II and Atg3, 5, 7, and 12 in adult rat annulus fibrosus cells treated with both high glucose concentrations compared to the control (A, B). The ratio of LC3-II/LC3-I expression also increased in a dose- and time-dependent manner (C). LC, light chain; Atg, autophagy-related gene. ${ }^{*} p<0.05 ;{ }^{* *} p<0.01$.

\section{Discussion}

The current findings demonstrate that both high glucose concentrations significantly increased the expressions of autophagy markers, such as beclin-1, LC3-II, Atg3, 5, 7 and 12, in adult rat NP and AF cells in a dose- respectively time-dependent manner. Also the ratio of LC3-II/LC3I expression was increased in adult rat NP and AF cells in a dose-respectively time-dependent manner. These results suggest that the autophagy of adult intervertebral disc cells may be a potential biochemical mechanism for the intervertebral disc degeneration in adult patients with diabetes mellitus. Thus, the prevention of autophagy in adult intervertebral disc cells can be considered as a therapeutic target to prevent or to delay the intervertebral disc degeneration in adult patients with diabetes mellitus.

Autophagy (literally self-eating) is a vital catabolic process by which cells engulf and breakdown intracellular proteins and organelles in the lysosome and repurposes the constituents for a new biosynthesis [14-16]. It continuously occurs under normal conditions as a method of quality control to remove and recycle damaged proteins and organelles. Autophagy is a crucial mechanism in the response to either extra- or intracellular stress and can result in cell survival under certain circumstances. However, an over-activation of autophagy may result in autophagic cell death, which differs from apoptosis and programmed necrosis [15,19-22]. The initiation and elongation of autophagy are mediated by ATGs and LC3 proteins. Beclin-1 is part of the class III PI3K complex that is required for the formation of the autophagic vesicle and the interference with beclin-1 prevents the autophagy induction $[17,18]$. However, there have been little studies to investigate the relationship between diabetes mellitus, autophagy of intervertebral disc cells and the intervertebral disc degeneration. Previously, two studies [22,23] just reported the autophagy of young notochordal cells, which correspond to the young human generation only. Thus, no information was available about the effect of diabetes mellitus on the autophagy of adult NP and AF cells. Moreover, Jiang et al. [22] did not investigate the expression of Atg genes, which are essential for the execution of autophagy. Thus, no information was available about the effect of diabetes mellitus on autophagy in adult NP and AF cells, which correspond to the adult human generation.

The intervertebral disc is a highly specialized structure that consists of an outer AF, that surrounds the inner 
NP. The NP undergoes a chronological transition from a notochordal to a fibrocartilaginous NP. This transition accompanies changes in cell types from young notochordal cells to so-called adult NP chondrocyte-like cells. In human, this transition is usually completed by the second decade [24]. In contrast to NP cells, AF cells are fibroblastic, which is phenotypically different, and don't undergo chronological changes with age. In addition, there are some differences in the composition of the extracellular matrix produced by NP and AF cells of young and adult humans. Thus, additional studies are needed to investigate whether there is any difference between young and adult NP and AF cells in terms of the effect of diabetes mellitus on autophagy. For this purpose, an in vitro cell culture study was conducted using NP and AF cells which were obtained from 24 -week-old adult rats.

This study demonstrated a dose- and time-dependent increase of expressions of autophagy markers, such as beclin-1, LC3, and ATG3, 5, 7 and 12 from culture day 1 to culture day 3 in both high glucose concentrations, when compared to a normal control group in adult rat NP and AF cells. In addition, the ratio of LC3-II/LC3-I expression also increased in a dose- and time-dependent manner. Thus, autophagy seems to be a potential biochemical mechanism for the intervertebral disc degeneration in adult patients with diabetes mellitus irrespective of the type of disc cells. Those results suggest that the prevention of autophagy might be a therapeutic target for the intervertebral disc degeneration in adult patients with diabetes mellitus.

The current study had some limitations. Firstly, the generation of oxidative stress, such as ROS in NP and AF cells of adult rats was not investigated. Secondly, an in vitro high glucose-induced diabetic model cannot perfectly reflect all aspects of the in vivo intervertebral disc degeneration in adult patients with diabetes mellitus, especially in terms of high glucose concentrations and culture period. Thus, further in vivo studies are needed to clarify these limitations.

\section{Conclusions}

The current study demonstrates the dose- and time-dependent effect of high glucose concentrations on autophagy in adult rat NP and AF cells. This results in excessive autophagy of adult intervertebral disc cells, which leads to an intervertebral disc degeneration. Thus, the preven- tion of autophagy in adult intervertebral disc cells might be considered to be a therapeutic target for the intervertebral disc degeneration in adult patients with diabetes mellitus.

\section{Conflict of Interest}

No potential conflict of interest relevant to this article was reported.

\section{References}

1. Alberti KG, Zimmet P, Shaw J. Metabolic syndrome: a new world-wide definition. A Consensus Statement from the International Diabetes Federation. Diabet Med 2006;23:469-80.

2. McClelland AD, Kantharidis P. microRNA in the development of diabetic complications. Clin Sci (Lond) 2014;126:95-110.

3. Mobbs RJ, Newcombe RL, Chandran KN. Lumbar discectomy and the diabetic patient: incidence and outcome. J Clin Neurosci 2001;8:10-3.

4. Sakellaridis N. The influence of diabetes mellitus on lumbar intervertebral disk herniation. Surg Neurol 2006;66:152-4.

5. Simpson JM, Silveri CP, Balderston RA, Simeone FA, An HS. The results of operations on the lumbar spine in patients who have diabetes mellitus. J Bone Joint Surg Am 1993;75:1823-9.

6. Won HY, Park JB, Park EY, Riew KD. Effect of hyperglycemia on apoptosis of notochordal cells and intervertebral disc degeneration in diabetic rats. J Neurosurg Spine 2009;11:741-8.

7. Park EY, Park JB. Dose- and time-dependent effect of high glucose concentration on viability of notochordal cells and expression of matrix degrading and fibrotic enzymes. Int Orthop 2013;37:1179-86.

8. Victor VM, Rocha M, Herance R, Hernandez-Mijares A. Oxidative stress and mitochondrial dysfunction in type 2 diabetes. Curr Pharm Des 2011;17:3947-58.

9. Baynes JW. Role of oxidative stress in development of complications in diabetes. Diabetes 1991;40:405-12.

10. Jung HS, Lee MS. Role of autophagy in diabetes and mitochondria. Ann N Y Acad Sci 2010;1201:79-83.

11. Nishikawa T, Araki E. Impact of mitochondrial ROS production in the pathogenesis of diabetes mellitus and its complications. Antioxid Redox Signal 2007; 
9:343-53.

12. Srinivas V, Bohensky J, Zahm AM, Shapiro IM. Autophagy in mineralizing tissues: microenvironmental perspectives. Cell Cycle 2009;8:391-3.

13. Hamasaki M, Yoshimori T. Where do they come from? Insights into autophagosome formation. FEBS Lett 2010;584:1296-301.

14. Xie Z, Klionsky DJ. Autophagosome formation: core machinery and adaptations. Nat Cell Biol 2007;9: 1102-9.

15. Wang SY, Yu QJ, Zhang RD, Liu B. Core signaling pathways of survival/death in autophagy-related cancer networks. Int J Biochem Cell Biol 2011;43:1263-6.

16. Furuya N, Yu J, Byfield M, Pattingre S, Levine B. The evolutionarily conserved domain of Beclin 1 is required for Vps34 binding, autophagy and tumor suppressor function. Autophagy 2005;1:46-52.

17. He C, Zhu H, Li H, Zou MH, Xie Z. Dissociation of Bcl-2-Beclin1 complex by activated AMPK enhances cardiac autophagy and protects against cardiomyocyte apoptosis in diabetes. Diabetes 2013;62:1270-81.

18. Chen ZF, Li YB, Han JY, et al. The double-edged effect of autophagy in pancreatic beta cells and diabetes. Autophagy 2011;7:12-6.

19. Carames B, Taniguchi N, Otsuki S, Blanco FJ, Lotz
M. Autophagy is a protective mechanism in normal cartilage, and its aging-related loss is linked with cell death and osteoarthritis. Arthritis Rheum 2010;62: 791-801.

20. Murrow L, Debnath J. Autophagy as a stress-response and quality-control mechanism: implications for cell injury and human disease. Annu Rev Pathol 2013;8:105-37.

21. Jing $\mathrm{K}, \mathrm{Lim} \mathrm{K}$. Why is autophagy important in human diseases? Exp Mol Med 2012;44:69-72.

22. Jiang L, Zhang X, Zheng X, et al. Apoptosis, senescence, and autophagy in rat nucleus pulposus cells: Implications for diabetic intervertebral disc degeneration. J Orthop Res 2013;31:692-702.

23. Park EY, Park JB. High glucose-induced oxidative stress promotes autophagy through mitochondrial damage in rat notochordal cells. Int Orthop 2013; 37:2507-14.

24. Kim KW, Lim TH, Kim JG, Jeong ST, Masuda K, An HS. The origin of chondrocytes in the nucleus pulposus and histologic findings associated with the transition of a notochordal nucleus pulposus to a fibrocartilaginous nucleus pulposus in intact rabbit intervertebral discs. Spine (Phila Pa 1976) 2003;28:98290. 